Supporting Information

\title{
Compatibilizing Immiscible Polymer Blends with Sparsely Grafted Nanoparticles
}

Husam Alkhodairi, ${ }^{\dagger}$ Sebastian T. Russell,${ }^{\dagger}$ Julia Pribyl,${ }^{\ddagger}$ Brian C. Benicewicz, ${ }^{\ddagger}$ and Sanat K. Kumar*, ${ }^{\dagger}$

$\dagger$ Department of Chemical Engineering, Columbia University, New York, New York 10027, United States

† Department of Chemistry, University of South Carolina, Columbia, South Carolina 29208, United States

*Correspondence to sk2794@ columbia.edu 


\section{Experimental Details}

Preparation of PS-g-SiO 2 : Polystyrene-grafted silica nanoparticles (PS-g-SiO ${ }_{2}$ ) were synthesized by reversible addition-fragmentation chain transfer (RAFT) polymerization starting with the chain-transfer agent (CTA) covalently tethered to the NP surface. The following is a brief description of the complete synthetic process. First, a solution of colloidal silica NPs (Nissan Chemicals Inc., 30 wt\% dispersion in methyl isobutyl ketone) was diluted with 50 vol\% tetrahydrofuran (THF) and 3-aminopropyldimethylmethoxysilane was added. The mixture was heated at $65{ }^{\circ} \mathrm{C}$ for $4 \mathrm{~h}$ under an inert $\left(\mathrm{N}_{2}\right)$ atmosphere. After cooling the reaction mixture to $0{ }^{\circ} \mathrm{C}$, the surface anchored amine groups were allowed to react with 2mercaptothiazoline-activated 4-cyanopentanoic acid (CPDB) overnight under an $\mathrm{N}_{2}$ blanket, coming to room temperature. After precipitation in an antisolvent (hexanes) and centrifugation, the graft density of tethered CTA was determined by UV-Vis spectroscopy in comparison to a calibration made from known amounts of free CTA in solution. The surface polymerization of styrene was performed in THF at $65{ }^{\circ} \mathrm{C}$ with azobisisobutyronitrile (AIBN) as an exogenous radical source. The PS-g-SiO particles were then precipitated in hexanes, recovered by centrifugation, and redispersed in THF. The CTA was cleaved from the chain ends (to prevent undesired side reactions) by adding a large excess of AIBN (40 eq.) and heating the mixture at reflux for $3 \mathrm{~h}$. 


\section{Imaging and Image Analysis}

Thin sections of the samples ( $\sim 60 \mathrm{~nm})$ were cut on a Leica EM UC6 ultramicrotome with a diamond knife and collected on carbon/formvar-coated copper grids (TEM) or glass coverslips (phase-contrast optical microscopy).

Multiple regions were imaged for each sample. ImageJ was used to quantify the droplet areas $A_{i}$, which were then converted into equivalent sphere diameters as $D_{i}=$ $\sqrt{4 A_{i} / \pi}$. A total of 400-1000 droplets were measured for each sample. The number average $\left(D_{n}\right)$ and volume average $\left(D_{v}\right)$ droplet diameters were calculated as follows ${ }^{1}$

$$
\begin{aligned}
& D_{n}=\frac{\sum n_{i} D_{i}}{\sum n_{i}} \\
& D_{v}=\frac{\sum n_{i} D_{i}^{4}}{\sum n_{i} D_{i}^{3}}
\end{aligned}
$$

where $n_{i}$ is the number of droplets having diameter $D_{i}$. The droplet size dispersity (PDI) was then calculated as $D_{v} / D_{n}$.

The $D_{n}$ values reported in the main text were multiplied by $4 / \pi$ to account for the systematic underestimation in $D_{i}$ originating from taking 2-dimensional cuts through the droplets (ultramicrotomy). ${ }^{2}$ 


\section{Interfacial Tension Estimation}

Table S1. Surface energy data for PMMA, PS, and silica at $150{ }^{\circ} \mathrm{C}$.

\begin{tabular}{|c|c|c|c|c|}
\hline Component & $\begin{array}{c}\boldsymbol{\gamma} \\
(\mathbf{d y n e} / \mathbf{c m})\end{array}$ & $\begin{array}{c}\boldsymbol{\gamma}^{\boldsymbol{d}} \\
(\mathbf{d y n e} / \mathbf{c m})\end{array}$ & $\begin{array}{c}\boldsymbol{\gamma}^{\boldsymbol{p}} \\
(\mathbf{d y n e} / \mathbf{c m})\end{array}$ & $\begin{array}{c}\boldsymbol{d} \boldsymbol{\gamma} / \boldsymbol{d T} \\
(\mathbf{d y n e} / \mathbf{c m ~ K})\end{array}$ \\
\hline PMMA $^{3,4}$ & 31.22 & 22.48 & 8.74 & -0.076 \\
\hline $\mathrm{PS}^{3,4}$ & 31.34 & 26.57 & 4.70 & -0.072 \\
\hline Silica $^{1}$ & 67.50 & 24.81 & 42.69 & -0.100 \\
\hline
\end{tabular}

The PMMA/silica $\left(\gamma_{1 C}\right)$, PS/silica $\left(\gamma_{2 C}\right)$, and PMMA/PS $\left(\gamma_{12}\right)$ interfacial tensions were estimated from surface energy data available in the literature (Table S1) using the Owens-Wendt method ${ }^{5}$

$$
\gamma_{i j}=\gamma_{i}+\gamma_{j}-2 \sqrt{\gamma^{d}{ }_{i} \gamma^{d}}-2 \sqrt{\gamma^{p}{ }_{i} \gamma^{p}{ }_{j}}
$$

where $\gamma_{i j}$ is the interfacial tension between components $i$ and $j$; and $\gamma_{i}$ and $\gamma_{j}$ are the total surface energy of components $i$ and $j$, respectively. The subscripts $d$ and $p$ denote the dispersive and polar components of the surface energy, respectively, such that $\gamma_{i}=\gamma_{i}^{d}+\gamma_{i}^{p}$. The surface energies were reported at room temperature in the literature and were converted to $150{ }^{\circ} \mathrm{C}$ using the reported temperature coefficients, $d \gamma / d T$ (Table S1). It was assumed that the dispersive and polar components have the same temperature dependence. Thus,

$$
\gamma_{1 C}=12.86 ; \gamma_{2 C}=19.18 ; \gamma_{12}=0.87 \mathrm{mN} / \mathrm{m}
$$




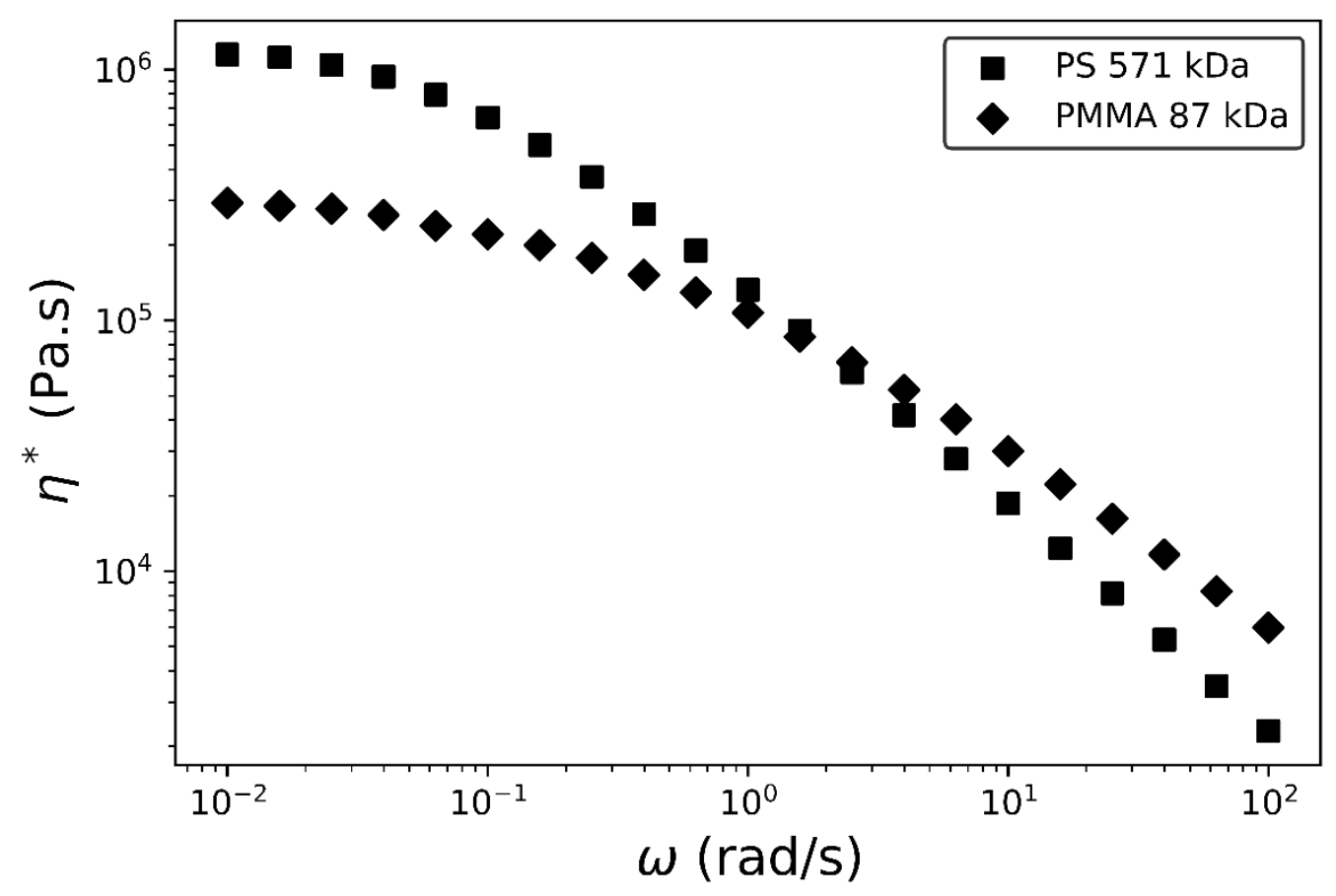

Figure S1. The complex viscosity $\left(\eta^{*}\right)$ as a function of the angular frequency $(\omega)$ for PS $\left(M_{w} \approx\right.$ $\left.571 \mathrm{kDa}, M_{w} / M_{n} \approx 1.04\right)$ and PMMA $\left(M_{w} \approx 87 \mathrm{kDa}, M_{w} / M_{n} \sim 2\right)$ at $190{ }^{\circ} \mathrm{C}$. The measurement was obtained at $1 \%$ strain and was followed by a strain sweep to ensure it lied within the linear viscoelastic regime. 


\section{References}

(1) Elias, L.; Fenouillot, F.; Majeste, J. C.; Cassagnau, P. Morphology and Rheology of Immiscible Polymer Blends Filled with Silica Nanoparticles. Polymer (Guildf). 2007, $48 \quad$ (20), $6029-6040$. https://doi.org/10.1016/j.polymer.2007.07.061.

(2) Wu, S. Phase Structure and Adhesion in Polymer Blends: A Criterion for Rubber Toughening. Polymer (Guildf). 1985, 26 (12), 1855-1863. https://doi.org/10.1016/0032-3861(85)90015-1.

(3) $\mathrm{Wu}, \mathrm{S}$. Surface and Interfacial Tensions of Polymer Melts. II. Poly(Methyl Methacrylate), Poly(n-Butyl Methacrylate), and Polystyrene. J. Phys. Chem. 1970, 74 (3), 632-638. https://doi.org/10.1021/j100698a026.

(4) Wu, S. Interfacial and Surface Tensions of Polymers. J. Macromol. Sci. Part $\begin{array}{llllll}C & \text { Polym. Rev. } & \mathbf{1 9 7 4}, & 10 & \text { (1), } & 1-73 .\end{array}$ https://doi.org/10.1080/15321797408080004.

(5) Owens, D. K.; Wendt, R. C. Estimation of the Surface Free Energy of Polymers. J. Appl. Polym. Sci. 1969, 13 (8), 1741-1747. https://doi.org/10.1002/app.1969.070130815. 